\title{
Ajuste de las distribuciones GVE, LOG y PAG con momentos L de orden mayor
}

\section{Fitting of GEV, GLO and GPA Distributions with Higher-Order L Moments}

\author{
Campos-Aranda Daniel Francisco \\ Profesor Jubilado de la Universidad Autónoma de San Luis Potosí \\ Correo:campos_aranda@hotmail.com
}

Información del artículo: recibido: marzo de 2015, aceptado: mayo de 2015

\section{Resumen}

El análisis probabilístico de datos hidrológicos extremos busca estimar predicciones confiables asociadas a altos periodos de retorno. Los momentos $\mathrm{LH}$ o de orden mayor, permiten caracterizar de una manera más eficiente la cola derecha de la función de distribución de probabilidades (FDP), al dar más importancia a los grandes valores de los datos. Usando los momentos LH se reduce la influencia indeseable que las magnitudes pequeñas de la muestra pueden tener en la estimación de las predicciones. En este trabajo se describe brevemente la teoría de los momentos L, como base para su generalización, lo cual conduce a los momentos LH, propuestos en 1997 por Wang Q.J. Se citan las ecuaciones que permiten estimar, con los métodos de momentos $\mathrm{L}$ y momentos LH, los tres parámetros de ajuste de las FDP: general de valores extremos (GVE), logística generalizada (LOG) y Pareto generalizada (PAG). Se realiza una aplicación numérica a los 21 registros disponibles de crecientes anuales de la Región Hidrológica Núm. 10 (Sinaloa), contrastando sus resultados con base en el error estándar de ajuste (EEA). El análisis de resultados mostró que la distribución PAG conduce a los mejores ajustes. También se observó que los momentos LH son una buena opción para abatir el EEA en las tres distribuciones utilizadas.

\section{Descriptores:}

- momentos L

- momentos LH o de orden mayor

- distribuciones GVE, LOG y PAG

- error estándar de ajuste

- Región Hidrológica Núm. 10 (Sinaloa) 


\begin{abstract}
The probabilistic analysis of extreme hydrological data aims to estimate reliable predictions associated with high return periods. The LH or higher-order moments can characterize in a more efficient way the right tail of the probability distribution function $(P D F)$, by giving more importance to large data values. The undesirable influence of the sample's small quantities is reduced by using the LH moments in the estimation of predictions. This paper briefly describes the theory of L moments, as a basis for its generalization, which leads to the LH moments, proposed in 1997 by Wang Q.J. By means of the $L$ and LH moments methods, the equations that allow the estimation of the three fitting parameters of the PDF: General Extreme Values (GEV), Generalized Logistics (GLO) and Generalized Pareto (GPA) are cited. A numerical application to the 21 available records annual floods of Hydrological Region No. 10 (Sinaloa) is performed, contrasting its results based on the standard error of fit (SEF). The analysis of results showed that the GPA distribution leads to the best fittings. It can be highlighted that $L H$ moments are a good choice to abate the SEF in the three distributions used.
\end{abstract}

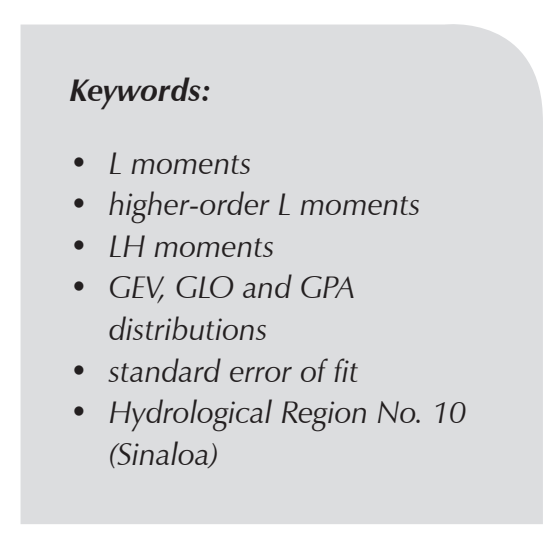

\section{Introducción}

La planeación, diseño y operación de las obras hidráulicas, se basa en las llamadas Crecientes de Diseño, cuya estimación lo más exacta posible, incrementa su seguridad hidrológica. El método más confiable para su estimación consiste en el análisis probabilístico de la información de gastos máximos anuales, a través del ajuste de una cierta función de distribución de probabilidades (FDP) y su uso posterior para realizar las predicciones buscadas. Esta técnica denominada análisis de frecuencia de crecientes (AFC) consta de cuatro etapas:

1) Recopilación de datos y verificación de su calidad estadística.

2) Selección de una FDP.

3) Elección de un método de estimación de sus parámetros de ajuste.

4) Verificación del ajuste logrado con cada FDP y técnica de estimación (Hosking y Wallis, 1997; Lee y Maeng, 2003; Gheidari, 2013).

En el AFC los periodos de retorno de diseño, comúnmente exceden varias veces la amplitud del registro disponible en años, entonces las predicciones buscadas son extrapolaciones que involucran dificultades y errores asociados. El error principal inherente a tales extrapolaciones radica en aceptar que la FDP adoptada y ajustada a los datos, es válida más allá de la magnitud máxima de los valores disponibles. Se han desarrollado dos enfoques para reducir el error citado; el primero consiste en utilizar FDP de gran flexibilidad, que están caracterizadas por más de tres parámetros de ajuste, como la distribución Kappa de cuatro parámetros y la Wakeby y las funciones mixtas de cinco parámetros. El segundo enfoque radica en seleccionar los datos de crecientes por utilizar, con la idea fundamental de dar más importancia a las observaciones grandes; se han propuesto tres técnicas:

a) La más antigua consiste en emplear crecientes superiores a un valor umbral.

b) Emplear muestras truncadas o censuradas.

c) Las más recientes, emplear técnicas de ajuste que eliminan datos pequeños, o bien, dan mayor influencia a los valores grandes, caso de los momentos L depurados y de orden mayor (Wang, 1997b; Moisello, 2007; Meshgi y Khalili, 2009a).

El objetivo de este trabajo consiste en exponer de manera breve la teoría de los momentos LH o de orden mayor y sus ventajas en el AFC, citando las ecuaciones que permiten estimar los tres parámetros de ajuste de las distribuciones GVE, LOG y PAG, con los métodos de momentos L y momentos LH. Estas FDP son de uso frecuente en los AFC. Se aplican ambas técnicas de ajuste a los 21 registros disponibles de crecientes anuales en la Región Hidrológica Núm. 10 (Sinaloa), revisando principalmente su homogeneidad hidrológica regional y después se evalúa el desempeño de las FDP mediante el error estándar de ajuste. Por último, se formulan las conclusiones del estudio.

\section{Resumen de la teoría operativa}

Momentos L poblacionales y de la muestra

Hosking y Wallis (1997) indican que los momentos L son un sistema alternativo que permite describir la forma de la FDP, cuyos antecedentes estadísticos nacieron 
con los momentos de probabilidad ponderada (MPP) de una variable aleatoria $X$ con función de probabilidades acumuladas $F(x)$, definidos por Greenwood et al. (1979) como las cantidades siguientes:

$$
M_{p, r, s}=\mathrm{E}\left\lfloor X^{p}\{F(x)\}^{r}\{1-F(x)\}^{s}\right\rfloor
$$

Particularmente útiles son los casos especiales: $\alpha_{r}=M_{1,0, r}$ y $\beta_{r}=M_{1, r, 0}$ cuyas expresiones son

$$
\alpha_{r}=\int_{0}^{1} x(F)(1-F)^{r} d F, \quad \beta_{r}=\int_{0}^{1} x(F) F^{r} d F
$$

donde $X=x(F)$ y $F=F(x)$. Las ecuaciones anteriores contrastan con la definición de los momentos ordinarios, que es

$$
\mathrm{E}\left(X^{r}\right)=\int_{0}^{1}[x(F)]^{r} d F
$$

Se observa que los momentos convencionales involucran potencias sucesivas de la llamada solución inversa $x(F)$, mientras que los MPP establecen potencias sucesivas de $(1-F)$ o de $F$ y ello se puede considerar como la integral de $x(F)$ ponderada por tales polinomios. Los MPP $\alpha_{r}$ y $\beta_{r}$ son la base de varios métodos que permiten estimar los parámetros de ajuste de una FDP. Sin embargo, de manera individual son difíciles de interpretar como medidas de escala y forma, pero tal información se obtiene con ciertas combinaciones lineales de ellos, que son precisamente los momentos $L(\lambda)$. Como medidas de ubicación, escala y forma (asimetría y curtosis), estos son respectivamente (Hosking y Wallis, 1997)

$$
\begin{aligned}
& \lambda_{1}=\alpha_{0}=\beta_{0} \\
& \lambda_{2}=\alpha_{0}-2 \alpha_{1}=2 \beta_{1}-\beta_{0} \\
& \lambda_{3}=\alpha_{0}-6 \alpha_{1}+6 \alpha_{2}=6 \beta_{2}-6 \beta_{1}+\beta_{0} \\
& \lambda_{4}=\alpha_{0}-12 \alpha_{1}+30 \alpha_{2}-20 \alpha_{3}=20 \beta_{3}-30 \beta_{2}+12 \beta_{1}-\beta_{0}
\end{aligned}
$$

Los cocientes de momentos $\mathrm{L}$ adimensionales se definen con similitud a los coeficientes de variación, asimetría y curtosis, como

$$
\begin{aligned}
& \tau_{2}=\lambda_{2} / \lambda_{1} \\
& \tau_{r}=\lambda_{r} / \lambda_{2} \quad \text { para } r=3 \mathrm{y} 4
\end{aligned}
$$

En una muestra de tamaño $n$, ordenada en forma progresiva, es decir que: $x_{1: n} \leq x_{2: n} \leq \ldots \leq x_{n: n}$, el estimador insesgado del MPP $\beta_{r}$ es (Hosking y Wallis, 1997)

$b_{r}=\frac{1}{n} \sum_{j=r+1}^{n} \frac{(j-1) \cdot(j-2) \cdots(j-r)}{(n-1) \cdot(n-2) \cdots(n-r)} x_{j: n} \quad$ para $r=0,1,2,3$

Entonces, las estimaciones con la muestra o serie de datos de los momentos L se obtienen con las ecuaciones 4 a 7 y se designan por $l_{r}$ para $r=1,2,3,4$ y sus cocientes son $t_{2}, t_{3}$ y $t_{4}$.

\section{Momentos L de orden mayor}

Desde que Hosking (1990) consolidó la teoría de los momentos L, estos se han empleado para analizar valores extremos de crecientes, vientos, lluvias, etcétera. Tales análisis estadísticos se orientan a predecir eventos extremos asociados a altos periodos de retorno, es decir, a bajas probabilidades de ser excedidos. Entonces, resulta relevante en estos análisis la cola derecha de la FDP y los datos extremos superiores de la muestra; surgiendo estas dudas: ¿Son los momentos L demasiado sensitivos a los valores bajos de la muestra? O bien, ¿Dan peso insuficiente a las grandes magnitudes de los datos? Para eliminar tales dudas, Wang (1997b) propone los momentos $\mathrm{L}$ de orden mayor $\left(\lambda^{\eta}\right)$, conocidos como "LH-moments" de higher que significa orden superior, como una generalización de los momentos L, que permiten una mejor caracterización de la cola derecha de la FDP y de los grandes eventos en los datos. Para ello, Wang (1997b) define los momentos de probabilidad ponderada normalizados, como

$B_{r}=\frac{\int_{0}^{1} x(F) F^{r} d F}{\int_{0}^{1} F^{r} d F}=(r+1) \int_{0}^{1} x(F) F^{r} d F=(r+1) \cdot \beta_{r}$

y entonces encuentra las expresiones siguientes para los momentos L de orden mayor

$$
\begin{aligned}
& \lambda_{1}^{\eta}=B_{\eta} \\
& \lambda_{2}^{\eta}=\frac{1}{2 !}(\eta+2)\left[B_{\eta+1}-B_{\eta}\right] \\
& \lambda_{3}^{\eta}=\frac{1}{3 !}(\eta+3)\left[(\eta+4) \cdot B_{\eta+2}-2(\eta+3) \cdot B_{\eta+1}+(\eta+2) \cdot B_{\eta}\right]
\end{aligned}
$$


$\lambda_{4}^{\eta}=\frac{1}{4 !}(\eta+4)\left[(\eta+6)(\eta+5) \cdot B_{\eta+3}-3(\eta+5)(\eta+4) \cdot B_{\eta+2}\right.$

$+3(\eta+4)(\eta+3) \cdot B_{\eta+1}-(\eta+3)$

Cuando $\eta=0$ los momentos LH son idénticos a los momentos L (ecuaciones 4 a 7). Conforme $\eta$ se incrementa, los momentos LH reflejan de mejor manera las características de la parte superior de la distribución y de los grandes eventos de los datos. Los momentos L también se han definido y estudiado como estadísticos de orden, al igual que los momentos L de orden mayor; en Wang (1997b) se puede consultar tal formulación. Los cocientes de momentos L de orden superior son

$\tau_{r}^{\eta}=\frac{\lambda_{r}^{\eta}}{\lambda_{2}^{\eta}} \quad$ para $r=3 \mathrm{y} 4$

\section{Ajuste de las distribuciones GVE, LOG y PAG con momentos $\mathrm{L}$}

Hosking y Wallis (1997) indican en su tabla 5.1, que cuando su parámetro de forma es negativo $(k<0)$ en estas FDP, tienen sus colas derechas más gruesas o densas (heavy tail) que las otras que son empleadas rutinariamente en los análisis de frecuencia de datos hidrológicos extremos; además tales FDP tienen una frontera superior cuando $k$ es positivo $(k>0)$ y cuando $k=0$ definen funciones de dos parámetros: la Gumbel, la Logística y la Exponencial, respectivamente.

El método de obtención de los parámetros de ubicación, escala y forma $(u, a$ y $k$ ) en las distribuciones GVE, LOG y PAG con base en los momentos L se puede consultar para cada una en: Stedinger et al. (1993), Rao y Hamed (2000) y Hosking y Wallis (1997). En la tabla 1 se enlistan para estas FDP: intervalo, fórmula matemática $F(x)$, solución inversa $x(F)$ y las ecuaciones del método de momentos L, para $k$, $a$ y $u$.

\section{Ajuste de las distribuciones GVE, LOG y PAG con momentos L de orden mayor}

Wang $(1997 a, b)$ desarrolló dos procedimientos para el ajuste de la distribución GVE con el método de los momentos L de orden mayor, el segundo de ellos, estima el parámetro de forma $(k)$ con base en el cociente $\tau_{3}^{\eta}$ y el

Tabla 1. Fórmulas matemáticas de las FDP indicadas y ecuaciones para estimar sus tres parámetros de ajuste $(k, a, u)$ con el método de momentos $\mathrm{L}$

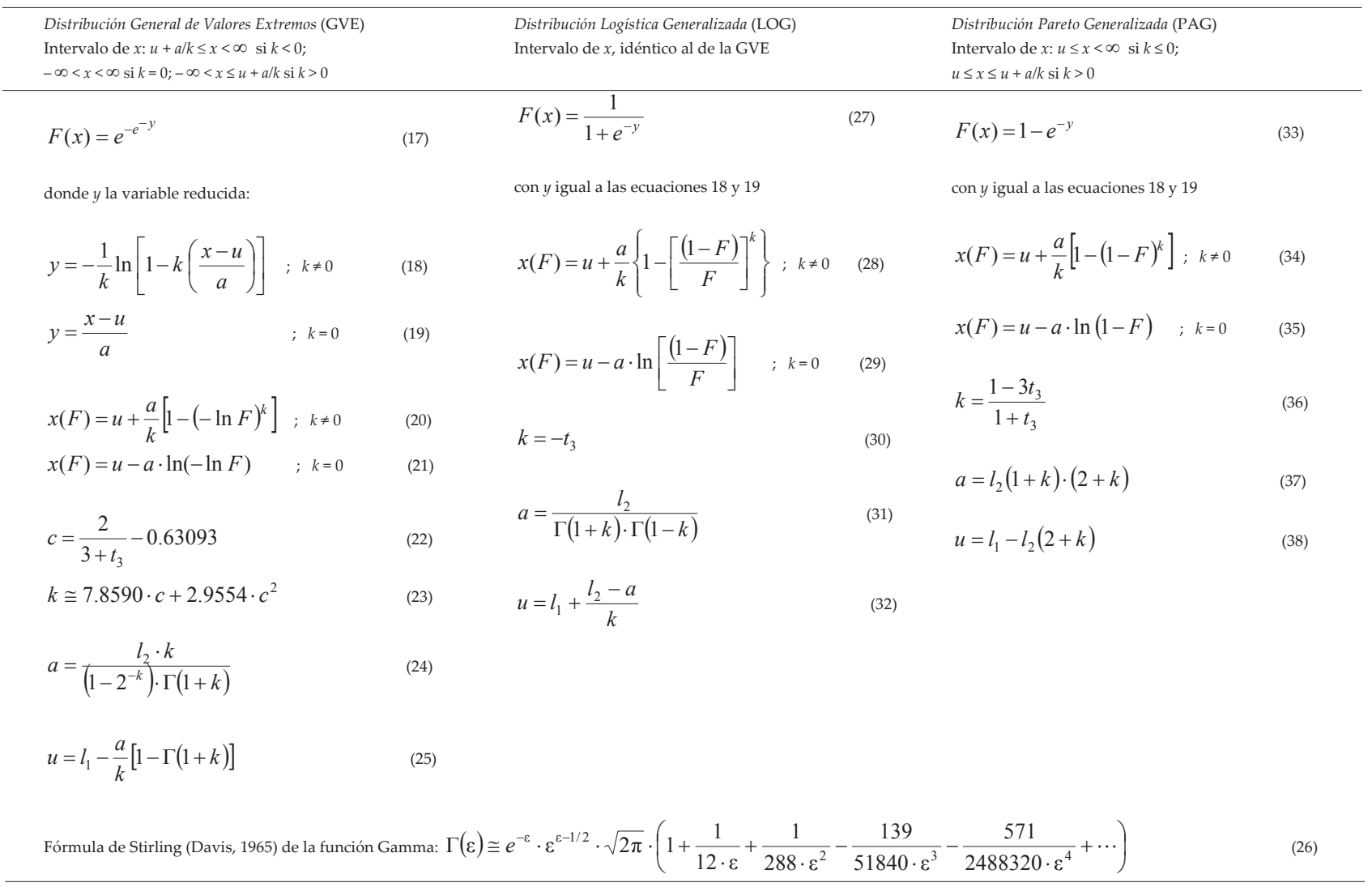


primero se parece al de los momentos $\mathrm{L}$ y es el siguiente

$c^{\eta}=\frac{(\eta+2) \cdot \beta_{\eta+1}-(\eta+1) \cdot \beta_{\eta}}{(\eta+3) \cdot \beta_{\eta+2}-(\eta+1) \cdot \beta_{\eta}}-\frac{\ln (\eta+2)-\ln (\eta+1)}{\ln (\eta+3)-\ln (\eta+1)}$

$k^{\eta} \cong a_{1} \cdot c^{\eta}+a_{2} \cdot\left(c^{\eta}\right)^{2}$

Los coeficientes $a_{1}$ y $a_{2}$ varían con el orden $\eta$ de los momentos $\mathrm{LH}$, tienen los valores de la tabla 2 y son aplicables en el intervalo: $-0.50 \leq k^{\eta} \leq 0.50$.

Tabla 2. Coeficientes $a_{1}$ y $a_{2}$ de la ecuación 40 y error absoluto máximo ( $\delta$ ) del parámetro de forma $k^{\eta}$ de la distribución GVE (Wang, 1997a)

\begin{tabular}{cccc}
\hline$\eta$ & $a_{1}$ & $a_{2}$ & $|\delta|$ \\
\hline 1 & 11.9082 & 2.7787 & $3.4 \cdot 10^{-4}$ \\
2 & 15.9316 & 2.7301 & $1.8 \cdot 10^{-4}$ \\
3 & 19.9455 & 2.7072 & $1.1 \cdot 10^{-4}$ \\
4 & 23.9546 & 2.6936 & $7.7 \cdot 10^{-5}$ \\
\hline
\end{tabular}

$a^{\eta}=\frac{k^{\eta}\left[(\eta+2) \cdot \beta_{\eta+1}-(\eta+1) \cdot \beta_{\eta}\right]}{\Gamma\left(1+k^{\eta}\right) \cdot\left[(\eta+1)^{-k^{\eta}}-(\eta+2)^{-k^{\eta}}\right]}$

$u^{\eta}=(\eta+1) \cdot \beta_{\eta}-\frac{a^{\eta}}{k^{\eta}}\left[1-\Gamma\left(1+k^{\eta}\right) \cdot(\eta+1)^{-k^{\eta}}\right]$

Meshgi y Khalili (2009b) obtuvieron las expresiones que permiten estimar los tres parámetros de ajuste de la distribución logística generalizada (LOG) con el método de los momentos $L$ de orden mayor, estas son

$k^{\eta}=-\frac{(\eta+3) \cdot(\eta+2) \cdot \beta_{\eta+2}-\left[(\eta+2)^{2}+(\eta+2) \cdot(\eta+1)\right] \beta_{\eta+1}+(\eta+1)^{2} \beta_{\eta}}{(\eta+2) \cdot \beta_{\eta+1}-(\eta+1) \cdot \beta_{\eta}}$

$a^{\eta}=\frac{\Gamma(\eta+2) \cdot\left[(\eta+2) \cdot \beta_{\eta+1}-(\eta+1) \cdot \beta_{\eta}\right]}{\Gamma\left(\eta+1-k^{\eta}\right) \cdot \Gamma\left(1+k^{\eta}\right)}$

$u^{\eta}=(\eta+1) \cdot \beta_{\eta}-\frac{a^{\eta}}{k^{\eta}}\left[1-\frac{\Gamma\left(\eta+1-k^{\eta}\right) \cdot \Gamma\left(1+k^{\eta}\right)}{\Gamma(\eta+1)}\right]$

Meshgi y Khalili (2009b) también desarrollaron las ecuaciones que permiten estimar los tres parámetros de ajuste de la distribución pareto generalizada (PAG) con el método de los momentos L de orden mayor, estas son

$$
k^{\eta}=-\frac{-5-2 \eta+\frac{(\eta+3) \cdot\left[(\eta+3) \cdot \beta_{\eta+2}-(\eta+1) \cdot \beta_{\eta}\right]}{(\eta+2) \cdot \beta_{\eta+1}-(\eta+1) \cdot \beta_{\eta}}}{-1+\frac{(\eta+3) \cdot \beta_{\eta+2}-(\eta+1) \cdot \beta_{\eta}}{(\eta+2) \cdot \beta_{\eta+1}-(\eta+1) \cdot \beta_{\eta}}}
$$

$a^{\eta}=-\frac{k^{\eta} \cdot \Gamma\left(\eta+3+k^{\eta}\right) \cdot \Gamma\left(\eta+2+k^{\eta}\right) \cdot\left[(\eta+2) \cdot \beta_{\eta+1}-(\eta+1) \cdot \beta_{\eta}\right]}{(\eta+1) ! \Gamma\left(1+k^{\eta}\right) \cdot\left[(\eta+2) \cdot \Gamma\left(\eta+2+k^{\eta}\right)-\Gamma\left(\eta+3+k^{\eta}\right)\right]}$

$$
u^{\eta}=(\eta+1) \cdot \beta_{\eta}-\frac{a^{\eta}}{k^{\eta}}\left[1-\frac{(\eta+1) \cdot \Gamma(\eta+1) \cdot \Gamma\left(1+k^{\eta}\right)}{\Gamma\left(\eta+2+k^{\eta}\right)}\right]
$$

\section{Diagramas de cocientes de momentos $\mathrm{L}$ de orden mayor}

Hosking y Wallis (1997) presentan la relación gráfica que tiene el cociente de momentos L de asimetría $\left(\tau_{3}\right)$ con el de curtosis $\left(\tau_{4}\right)$ en cinco FDP, lo cual constituye el llamado Diagrama de cocientes de momentos L. Tal gráfica se puede utilizar para seleccionar la mejor FDP, de acuerdo con los valores $t_{3}$ y $t_{4}$ de la muestra local o a su estimación regional. Las relaciones gráficas para las distribuciones GVE, LOG y PAG son polinomios de grado $(p)$ sexto, segundo y cuarto, cuya ecuación general es

$\tau_{4}=\sum_{p=0}^{6} d_{p} \cdot \tau_{3}^{p}$

Los coeficientes $d_{p}$ de la ecuación 49 se tienen en la tabla 3 ; tal ecuación tiene una aproximación de \pm 0.0005 cuando $-0.60 \leq \tau_{3} \leq 0.90$.

Tabla 3. Coeficientes de los polinomios de aproximación de $\tau_{4}$ en función de $\tau_{3}$ en las distribuciones GVE, LOG y PAG con momentos L, según Hoskin y Wallis (1997)

\begin{tabular}{cccc}
\hline Coef. & GVE & LOG & PAG \\
\hline$d_{0}$ & 0.10701 & 0.16667 & - \\
$d_{1}$ & 0.11090 & - & 0.20196 \\
$d_{2}$ & 0.84838 & 0.83333 & 0.95924 \\
$d_{3}$ & -0.06669 & - & -0.20096 \\
$d_{4}$ & 0.00567 & - & 0.04061 \\
$d_{5}$ & -0.04208 & - & - \\
$d_{6}$ & 0.03763 & - & - \\
\hline
\end{tabular}


Para obtener los diagramas de momentos L de orden mayor, habrá que encontrar la relación entre $\tau_{3}^{\eta} \mathrm{y} \tau_{4}^{\eta}$ para cada FDP. Wang (1997b) desarrolló tal relación para la distribución GVE, al obtener sus expresiones de $\lambda_{2}^{\eta}, \lambda_{3}^{\eta} \mathrm{y}$ $\lambda_{4}^{\eta}$. Meshgi y Khalili (2009a) la exponen en forma de polinomio de grado cuarto, con sus coeficientes $e_{p}$ mostrados en la tabla 4; además, desarrollan y presentan en forma similar tal relación para las distribuciones LOG y PAG, cuyos coeficientes $f_{p}$ y $g_{p}$, también se citan en la tabla 4.

\section{Medida cuantitativa del ajuste}

A mediados de la década de los años setenta se estableció el error estándar de ajuste (EEA) como un indicador estadístico cuantitativo que estima la calidad del ajuste y que además permite la comparación objetiva entre las diversas FDP que se ajustan a una muestra, ya que tiene las unidades de los datos. Su expresión es la siguiente (Kite, 1977)

$$
E E A=\sqrt{\frac{\sum_{i=1}^{n}\left(x_{i}-\hat{x}_{i}\right)^{2}}{n-n p}}
$$

en donde, $n$ y $n p$ son el número de datos de la muestra y de parámetros de ajuste, en este caso tres; $x_{i}$ son los datos ordenados de menor a mayor y $\hat{x}_{i}$ son los valores estimados con la solución inversa $x(F)$ para una probabilidad de no excedencia, estimada con la fórmula de Weibull (Benson, 1962)

$$
P(X<x)=\frac{m}{n+1}
$$

donde $m$ es el número de orden del dato, con 1 para el menor y $n$ para el mayor.

\section{Desarrollo del contraste numérico}

Registros de crecientes anuales procesados

Fueron 21 registros de gastos máximos anuales instantáneos $\left(\mathrm{m}^{3} / \mathrm{s}\right)$ de las estaciones hidrométricas de la Región Hidrológica Núm. 10 (Sinaloa) que no tienen régimen de escurrimiento afectado por embalses o cambios drásticos en sus cuencas y que cuentan con más de 20 años de datos en el sistema BANDAS (IMTA, 2002). Los registros de las estaciones de aforos Huites y Guamuchil, llegan hasta el año en que la construcción del embalse respectivo afectó su escurrimiento. Los datos de la estación San Francisco se tomaron del Boletín Hidrológico Núm. 36 (SRH, 1975). Campos-Aranda (2014) verificó la calidad estadística de los registros citados y presenta sus momentos y cocientes L; además expone un mapa con la localización de las 21 estaciones hidrométricas y sus cuencas respectivas.

En las primeras cuatro columnas de la tabla 5 se citan las características generales de las 21 estaciones hidrométricas, expuestas en orden decreciente de tamaño de cuenca y en sus tres últimas columnas se tienen los valores de los cocientes de momentos L. Se observa que el registro más corto tiene 19 años y los más largos 56, con un valor mediano de 33 años.

\section{Verificación de la homogeneidad regional}

Se realizaron dos pruebas estadísticas a los 21 registros disponibles, la primera fue el cálculo de las Discordancias (Hosking y Wallis, 1997; Campos-Aranda, 2010), basada en los cocientes $t_{2}, t_{3}$ y $t_{4}$ expuestos en la tabla 5 , encontrado que ninguna muestra o serie de crecientes excede el valor crítico de $D c=3.00$, por lo cual no existen registros discordantes. Los dos registros con mayor discordancia fueronn La Tina $(D=2.35)$ y La Huerta $(D=2.13)$.

Tabla 4. Coeficientes de los polinomios de aproximación de $\tau_{4}^{\eta}$ en función de $\tau_{3}^{\eta}$ (ecuación 49) en las distribuciones GVE, LOG y PAG con momentos L de orden mayor, según Meshgi y Khalili (2009a)

\begin{tabular}{cccccccccccccc}
\hline & \multicolumn{9}{c}{ GVE } & \multicolumn{9}{c}{ LOG } & \multicolumn{3}{c}{ PAG } \\
\cline { 2 - 14 } & $e_{0}$ & $e_{1}$ & $e_{2}$ & $e_{3}$ & $e_{4}$ & $f_{0}$ & $f_{1}$ & $f_{2}$ & $g_{1}$ & $g_{2}$ & $g_{3}$ & $g_{4}$ \\
\hline 1 & 0.0666 & 0.1208 & 0.8711 & -0.0484 & 0.0084 & 0.1167 & 0.0187 & 0.8859 & 0.2083 & 0.9115 & -0.1134 & 0.0124 \\
2 & 0.0483 & 0.1357 & 0.8710 & -0.0317 & 0.0045 & 0.0889 & 0.0467 & 0.8960 & 0.2143 & 0.8816 & -0.0754 & 0.0059 \\
3 & 0.0378 & 0.1491 & 0.8644 & -0.0222 & 0.0026 & 0.0714 & 0.0714 & 0.8929 & 0.2187 & 0.8813 & -0.0538 & 0.0031 \\
4 & 0.0310 & 0.1602 & 0.8564 & -0.0163 & 0.0017 & 0.0595 & 0.0918 & 0.8856 & 0.2212 & 0.8374 & -0.0665 & -0.0112 \\
\hline
\end{tabular}


Tabla 5. Características generales de las 21 estaciones hidrométricas procesadas de la Región Hidrológica Núm. 10 (Sinaloa) y sus cocientes de momentos $\mathrm{L}$ de sus registros de crecientes anuales

\begin{tabular}{|c|c|c|c|c|c|c|}
\hline Núm. & $\begin{array}{c}\text { Estación } \\
\text { hidrométrica }\end{array}$ & $\begin{array}{c}\text { Área de } \\
\text { cuenca }\left(\mathrm{km}^{2}\right)\end{array}$ & $\begin{array}{l}\text { Años de } \\
\text { registro }(n)\end{array}$ & $t_{2}$ & $t_{3}$ & $t_{4}$ \\
\hline 1 & Huites & 26,057 & 1942-1992 (51) & 0.45868 & 0.49086 & 0.29757 \\
\hline 2 & San Francisco & 17,531 & 1941-1973 (33) & 0.41718 & 0.42671 & 0.22567 \\
\hline 3 & Santa Cruz & 8,919 & 1944-2002 (52) & 0.48122 & 0.42451 & 0.35919 \\
\hline 4 & Jaina & 8,179 & $1942-1998$ (56) & 0.47214 & 0.47970 & 0.34935 \\
\hline 5 & Palo Dulce & 6,439 & 1958-1986 (21) & 0.44948 & 0.61164 & 0.52064 \\
\hline 6 & Ixpalino & 6,166 & 1953-1999 (45) & 0.39951 & 0.44258 & 0.35056 \\
\hline 7 & La Huerta & 6,149 & 1970-1999 (28) & 0.34609 & 0.05350 & -0.00480 \\
\hline 8 & Chinipas & 5,098 & 1965-2002 (24) & 0.35652 & 0.29398 & 0.15342 \\
\hline 9 & Tamazula & 2,241 & 1963-1999 (32) & 0.30427 & 0.36007 & 0.29546 \\
\hline 10 & Naranjo & 2,064 & 1939-1984 (45) & 0.52382 & 0.40967 & 0.20800 \\
\hline 11 & Acatitán & 1,884 & 1955-2002 (43) & 0.52046 & 0.35069 & 0.22836 \\
\hline 12 & Guamuchil & 1,645 & 1940-1971 (32) & 0.42828 & 0.41560 & 0.28834 \\
\hline 13 & Choix & 1,403 & 1956-2002 (38) & 0.39541 & 0.42478 & 0.32621 \\
\hline 14 & Badiraguato & 1,018 & 1974-1999 (26) & 0.60699 & 0.64257 & 0.46378 \\
\hline 15 & El Quelite & 835 & 1961-2001 (33) & 0.45555 & 0.32149 & 0.16739 \\
\hline 16 & Zopilote & 666 & 1939-2001 (56) & 0.45227 & 0.20325 & 0.06008 \\
\hline 17 & Chico Ruiz & 391 & 1977-2002 (19) & 0.42017 & 0.10437 & -0.01184 \\
\hline 18 & El Bledal & 371 & 1938-1994 (56) & 0.42858 & 0.38055 & 0.27257 \\
\hline 19 & Pericos & 270 & 1961-1992 (30) & 0.34244 & 0.24867 & 0.14079 \\
\hline 20 & La Tina & 254 & $1960-1983(24)$ & 0.57488 & 0.48710 & 0.41474 \\
\hline 21 & Bamícori & 223 & 1951-1983 (33) & 0.48125 & 0.38784 & 0.15883 \\
\hline \multicolumn{4}{|c|}{ Valores ponderados sin incluir a la estación La Huerta: } & - & 0.39558 & 0.26291 \\
\hline
\end{tabular}

La segunda prueba aplicada fue el nuevo Test de Langbein (Fill y Stedinger, 1995; Campos-Aranda, 2012) donde se encuentra, que cuatro estaciones o registros quedan fuera de sus curvas de control, estas son: La Huerta, Tamazula, Chinipas y Pericos. Se eliminó la primera, ya que también fue de las más discordantes y se repitió la prueba; nuevamente aparecen las tres restantes como estaciones que se deben eliminar, pero tal número es el permitido en una región con 20 estaciones y confiabilidad de $95 \%$. Entonces se acepta la homogeneidad regional con los 20 registros de la tabla 5, al eliminar el de la estación La Huerta (Núm. 7).

En Bhuyan et al. (2010) se puede consultar un AFC regional completo, ya que la prueba de discordancias y la verificación de la homogeneidad se realiza con cada orden $(\eta)$ de los momentos L de orden mayor, así como la selección de la mejor FDP. En cambio, este trabajo se orientó al estudio de los ajustes locales, a través del EEA (ecuación 50).

\section{Cocientes de momentos $L$ de orden mayor}

Inicialmente, en las ecuaciones 12 a 15 , se sustituyó a $B_{\eta}$ por $\beta_{\eta}$ con base en la ecuación 11 y después al aplicar la ecuación 10 se obtuvieron los valores de los momentos L de orden mayor de cada muestra y con ellos los cocientes de asimetría y curtosis según la ecuación 16. Estos valores se muestran en la tabla 6.

\section{Selección de la FDP regional}

Principalmente se obtienen las magnitudes regionales representativas de los cocientes de momentos L originales y de orden mayor, por medio de sus valores ponderados con la amplitud de cada registro en años, a través de la expresión

$\tau_{r}^{\eta}=\sum_{i=1}^{20}\left[n_{i} \cdot\left(\tau_{r}^{\eta}\right)_{i} / \sum_{i=1}^{20} n_{i}\right]$ para $r=3,4$ 
Tabla 6. Cocientes de momentos $\mathrm{L}$ de orden mayor en los 20 registros de crecientes anuales de las estaciones hidrométricas indicadas de la Región Hidrológica Núm. 10 (Sinaloa)

\begin{tabular}{|c|c|c|c|c|c|c|c|c|c|}
\hline Núm. & $\begin{array}{c}\text { Estación } \\
\text { hidrométrica }\end{array}$ & $t_{3}^{1}$ & $t_{4}^{1}$ & $t_{3}^{2}$ & $t_{4}^{2}$ & $t_{3}^{3}$ & $t_{4}^{3}$ & $t_{3}^{4}$ & $t_{4}^{4}$ \\
\hline 1 & Huites & 0.47008 & 0.24687 & 0.44069 & 0.20961 & 0.41314 & 0.17903 & 0.38765 & 0.15323 \\
\hline 2 & San Francisco & 0.40646 & 0.19713 & 0.38603 & 0.17697 & 0.66831 & 0.16132 & 0.35286 & 0.14850 \\
\hline 3 & Santa Cruz & 0.48902 & 0.37575 & 0.51583 & 0.35295 & 0.51624 & 0.32348 & 0.50565 & 0.29751 \\
\hline 4 & Jaina & 0.49803 & 0.32271 & 0.49156 & 0.30048 & 0.48087 & 0.28636 & 0.47146 & 0.27860 \\
\hline 5 & Palo Dulce & 0.62450 & 0.51237 & 0.62897 & 0.50471 & 0.63084 & 0.49996 & 0.63224 & 0.49596 \\
\hline 6 & Ixpalino & 0.48872 & 0.32730 & 0.49040 & 0.30945 & 0.48447 & 0.29781 & 0.47821 & 0.28938 \\
\hline 8 & Chinipas & 0.30733 & 0.13645 & 0.30220 & 0.15755 & 0.31026 & 0.18009 & 0.32456 & 0.20767 \\
\hline 9 & Tamazula & 0.42843 & 0.31947 & 0.46220 & 0.34059 & 0.48566 & 0.35088 & 0.50097 & 0.35463 \\
\hline 10 & Naranjo & 0.38948 & 0.17092 & 0.36362 & 0.13954 & 0.33737 & 0.11800 & 0.31431 & 0.10604 \\
\hline 11 & Acatitán & 0.38107 & 0.23252 & 0.39379 & 0.22502 & 0.39588 & 0.22510 & 0.39731 & 0.23406 \\
\hline 12 & Guamuchil & 0.44202 & 0.27238 & 0.44252 & 0.27310 & 0.44419 & 0.28442 & 0.45050 & 0.30154 \\
\hline 13 & Choix & 0.46853 & 0.32997 & 0.48396 & 0.32760 & 0.48987 & 0.32303 & 0.49160 & 0.31727 \\
\hline 14 & Badiraguato & 0.59871 & 0.40610 & 0.56909 & 0.37309 & 0.54868 & 0.35175 & 0.53382 & 0.33796 \\
\hline 15 & El Quelite & 0.32884 & 0.18152 & 0.33952 & 0.18959 & 0.34836 & 0.18936 & 0.35284 & 0.18185 \\
\hline 16 & Zopilote & 0.19453 & 0.06582 & 0.19260 & 0.05832 & 0.18675 & 0.04521 & 0.17642 & 0.03267 \\
\hline 17 & Chico Ruiz & 0.07449 & -0.00637 & 0.05869 & 0.00979 & 0.05670 & 0.02939 & 0.06484 & 0.03954 \\
\hline 18 & El Bledal & 0.42053 & 0.27581 & 0.43521 & 0.27053 & 0.43930 & 0.26364 & 0.43891 & 0.25782 \\
\hline 19 & Pericos & 0.27725 & 0.15614 & 0.29725 & 0.14849 & 0.30265 & 0.13037 & 0.29724 & 0.10762 \\
\hline 20 & La Tina & 0.53906 & 0.45577 & 0.57457 & 0.47677 & 0.59727 & 0.49085 & 0.61332 & 0.50170 \\
\hline 21 & Bamícori & 0.35013 & 0.10438 & 0.30665 & 0.06683 & 0.26697 & 0.04162 & 0.23276 & 0.02749 \\
\hline \multicolumn{2}{|c|}{ Valores ponderados } & 0.41101 & 0.25269 & 0.41099 & 0.24128 & 0.41934 & 0.23156 & 0.39998 & 0.22441 \\
\hline
\end{tabular}
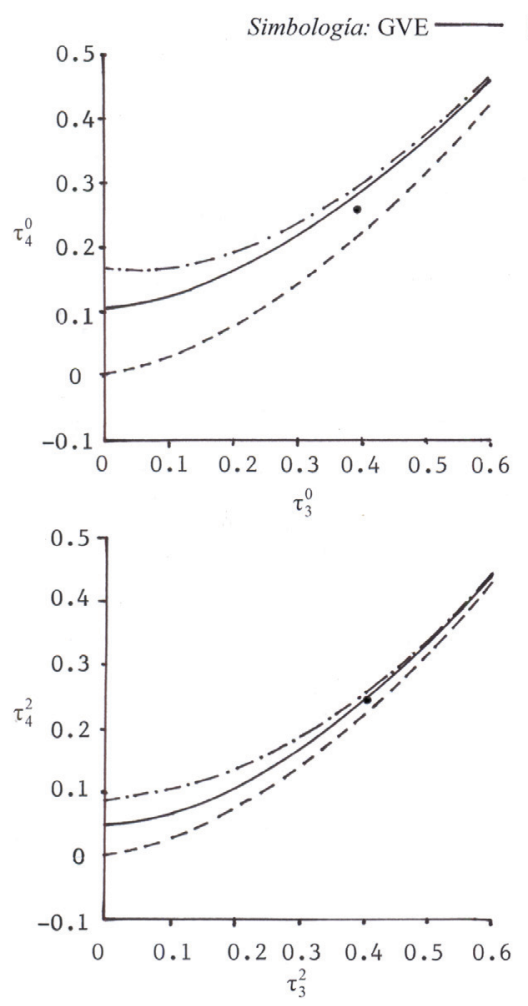

LOG -.-.- y PAG -.--
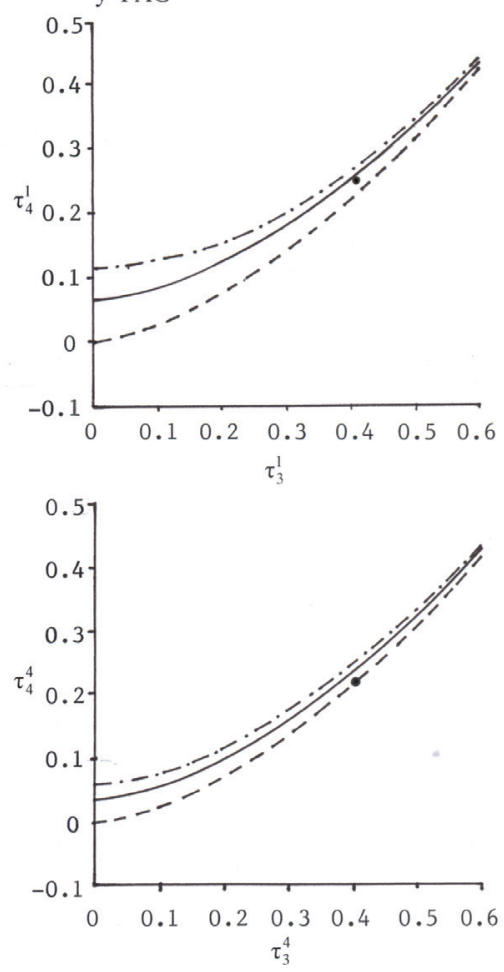

Figura 1. Selección de la FDP regional en los diagramas de cocientes de momentos LH 
donde

$i=$ contador de registros procesados $\mathrm{y}$

$n_{i}=$ su tamaño en años.

Los valores ponderados de los cocientes de momentos $\mathrm{L}$ y de los de orden mayor se tienen en los últimos renglones de las tablas 5 y 6 . Con base en la ecuación 49 se han construido los diagramas de cocientes de momentos mostrados en la figura 1, donde se dibujaron los valores ponderados de $\tau_{r}^{\eta}$; se observa que la FDP adecuada es la GVE y únicamente con $\eta=4$ es la PAG.

\section{Errores estándar de ajuste}

Con base en los momentos L y sus cocientes expuestos por Campos-Aranda (2014), se obtuvieron los tres parámetros de ajuste de las distribuciones GVE, LOG y PAG mediante las ecuaciones de la tabla 1. En segui$\mathrm{da}$, haciendo uso de las soluciones inversas, ecuaciones 20, 28 y 34, se obtuvieron las estimaciones $\hat{x}_{i}$ necesarias para aplicar la ecuación 50 del EEA. Los resultados se concentraron en la tabla 7, en las columnas indicadas con "L0".

De manera semejante, pero empleando los valores de $\beta_{r}$ calculados con la ecuación 10 , se estimaron los tres parámetros de ajuste de las funciones GVE, LOG y PAG, a través de las ecuaciones 39 a 48, para cada valor

Tabla 7. Errores estándar de ajuste $\left(\mathrm{m}^{3} / \mathrm{s}\right)$ obtenidos con los métodos de momentos $L$ y de momentos $L$ de orden mayor al ajustar las distribuciones GVE, LOG y PAG a los registros de crecientes anuales de las 20 estaciones hidrométricas indicadas de la Región Hidrológica Núm. 10 (Sinaloa)

\begin{tabular}{|c|c|c|c|c|c|c|c|c|c|c|c|c|c|c|c|c|c|}
\hline \multirow[b]{3}{*}{ Núm. } & \multirow{3}{*}{$\begin{array}{c}\text { Estación } \\
\text { Hidrométrica }\end{array}$} & \multicolumn{15}{|c|}{ Error estándar de ajuste (EEA) } & \multirow{3}{*}{$\begin{array}{c}E R \text { del } \\
E E A \\
(\%)\end{array}$} \\
\hline & & \multicolumn{5}{|c|}{ GVE } & \multicolumn{5}{|c|}{ LOG } & \multicolumn{5}{|c|}{ PAG } & \\
\hline & & L0 & L1 & L2 & L3 & L4 & L0 & L1 & L2 & L3 & L4 & L0 & L1 & $\mathrm{L} 2$ & L3 & L4 & \\
\hline 1 & Huites & 979 & 917 & $(892)$ & 974 & 1203 & 1039 & $(976)$ & 976 & 1133 & 1497 & 834 & 812 & [774] & 775 & 864 & 26.1 \\
\hline 2 & San Francisco & 377 & $(348)$ & 357 & 406 & 493 & 411 & $(385)$ & 416 & 514 & 668 & 302 & 290 & [282] & 286 & 308 & 36.5 \\
\hline 3 & Santa Cruz & $(406)$ & 408 & 424 & 428 & 417 & $(417)$ & 419 & 432 & 433 & 421 & 409 & [395] & 418 & 429 & 421 & 5.8 \\
\hline 4 & Jaina & 360 & 361 & 358 & 354 & (353) & 375 & 374 & 370 & (368) & 371 & 346 & 342 & 343 & 340 & [ 337] & 9.2 \\
\hline 5 & Palo Dulce & (895) & 920 & 947 & 970 & 990 & $(907)$ & 927 & 951 & 972 & 991 & [866] & 902 & 939 & 968 & 992 & 4.7 \\
\hline 6 & Ixpalino & 346 & 351 & 351 & 346 & (342) & 362 & 365 & 362 & 357 & (353) & 334 & [333] & 340 & 341 & 338 & 6.0 \\
\hline 8 & Chinipas & 139 & (136) & 143 & 146 & 141 & 152 & (151) & 175 & 196 & 195 & 126 & 127 & [126] & 128 & 135 & 19.8 \\
\hline 9 & Tamazula & (145) & 146 & 150 & 160 & 170 & 149 & (149) & 153 & 160 & 168 & 147 & [143] & 151 & 163 & 176 & 4.2 \\
\hline 10 & Naranjo & 156 & $(147)$ & 161 & 209 & 282 & 171 & (164) & 195 & 278 & 403 & 127 & 123 & [121] & 122 & 178 & 35.5 \\
\hline 11 & Acatitán & (227) & 228 & 228 & 228 & 229 & (236) & 239 & 241 & 247 & 253 & 231 & [223] & 225 & 227 & 229 & 5.8 \\
\hline 12 & Guamuchil & 235 & 234 & (233) & 233 & 234 & 244 & 243 & 241 & 240 & (240) & 225 & [225] & 226 & 229 & 234 & 6.7 \\
\hline 13 & Choix & 105 & (105) & 107 & 109 & 110 & 109 & (109) & 110 & 111 & 111 & 101 & [101] & 105 & 109 & 112 & 7.9 \\
\hline 14 & Badiraguato & 1077 & 1039 & 1007 & 985 & (971) & 1094 & 1056 & 1023 & 1001 & (990) & 1037 & 1000 & 977 & 958 & [943] & 5.0 \\
\hline 15 & El Quelite & 8 & (86) & 87 & 87 & 88 & (97) & 98 & 106 & 113 & 121 & 74 & 82 & [73] & 77 & 80 & 32.9 \\
\hline 16 & Zopilote & (48) & 5 & 74 & 99 & 134 & $(60)$ & 78 & 129 & 192 & 274 & [25] & 25 & 26 & 28 & 36 & 140.0 \\
\hline 17 & Chico Ruiz & (28) & 35 & 58 & 76 & 85 & (34) & 51 & 99 & 150 & 187 & [16] & 17 & 21 & 22 & 19 & 112.5 \\
\hline 18 & El Bledal & 64 & 64 & 64 & (64) & 64 & (67) & 67 & 67 & 67 & 67 & 68 & [63] & 64 & 66 & 66 & 6.3 \\
\hline 19 & Pericos & 29 & (28) & 28 & 29 & 183 & (33) & 34 & 37 & 44 & 57 & {$[24]$} & 25 & 27 & 27 & 26 & 37.5 \\
\hline 20 & La Tina & (90) & 92 & 96 & 102 & 107 & (92) & 93 & 97 & 102 & 107 & [87] & 90 & 96 & 102 & 108 & 5.7 \\
\hline 21 & Bamícori & 52 & $(48)$ & 57 & 86 & 125 & 56 & (54) & 71 & 116 & 185 & 43 & [40] & 43 & 54 & 74 & 35.0 \\
\hline \multicolumn{2}{|c|}{ Núm. de Mínimos } & 7 & 7 & 2 & 1 & 3 & 9 & 7 & - & 1 & 3 & 5 & 8 & 5 & - & 2 & - \\
\hline
\end{tabular}


Tabla 8. Predicciones $\left(\mathrm{m}^{3} / \mathrm{s}\right)$ obtenidas con la distribución Pareto Generalizada en los 20 registros de crecientes anuales de las estaciones hidrométricas indicadas de la Región Hidrológica Núm. 10 (Sinaloa)

\begin{tabular}{|c|c|c|c|c|c|c|c|c|c|}
\hline \multirow[b]{2}{*}{ Núm. } & \multirow{2}{*}{$\begin{array}{l}\text { Estación } \\
\text { Hidrométrica }\end{array}$} & \multirow{2}{*}{$\begin{array}{l}\text { Método de } \\
\text { ajuste }\end{array}$} & \multicolumn{7}{|c|}{ Periodos de retorno en años } \\
\hline & & & 5 & 10 & 25 & 50 & 100 & 500 & 1000 \\
\hline 1 & Huites & L2 & 4612 & 6968 & 10794 & 14343 & 18578 & 31836 & 39448 \\
\hline 2 & San Francisco & $\mathrm{L} 2$ & 2483 & 3566 & 5155 & 6487 & 7944 & 11869 & 13826 \\
\hline 3 & Santa Cruz & L1 & 1395 & 2097 & 3320 & 4536 & 6077 & 11435 & 14820 \\
\hline 4 & Jaina & $\mathrm{L} 4$ & 1350 & 2060 & 3301 & 4538 & 6108 & 11588 & 15061 \\
\hline 5 & Palo Dulce & L0 & 1288 & 1984 & 3386 & 4988 & 7282 & 17183 & 24745 \\
\hline 6 & Ixpalino & L1 & 1541 & 2223 & 3411 & 4591 & 6087 & 11282 & 14562 \\
\hline 8 & Chinipas & L2 & 1292 & 1712 & 2233 & 2602 & 2952 & 3694 & 3985 \\
\hline 9 & Tamazula & L1 & 765 & 1020 & 1421 & 1782 & 2201 & 3454 & 4143 \\
\hline 10 & Naranjo & L2 & 1010 & 1507 & 2203 & 2763 & 3352 & 4843 & 5543 \\
\hline 11 & Acatitán & L1 & 1272 & 1869 & 2734 & 3449 & 4222 & 6264 & 7262 \\
\hline 12 & Guamuchil & L1 & 966 & 1402 & 2105 & 2752 & 3519 & 5891 & 7238 \\
\hline 13 & Choix & L1 & 457 & 655 & 988 & 1308 & 1703 & 3007 & 3794 \\
\hline 14 & Badiraguato & L4 & 1474 & 2586 & 4774 & 7220 & 10654 & 24957 & 35555 \\
\hline 15 & El Quelite & L2 & 756 & 1054 & 1454 & 1760 & 2070 & 2801 & 3122 \\
\hline 16 & Zopilote & L0 & 596 & 777 & 961 & 1068 & 1153 & 1291 & 1332 \\
\hline 17 & Chico Ruiz & L0 & 353 & 429 & 490 & 518 & 537 & 558 & 562 \\
\hline 18 & El Bledal & L1 & 407 & 584 & 859 & 1103 & 1383 & 2203 & 2645 \\
\hline 19 & Pericos & L0 & 374 & 480 & 599 & 676 & 742 & 865 & 906 \\
\hline 20 & La Tina & L0 & 150 & 239 & 392 & 539 & 722 & 1335 & 1709 \\
\hline 21 & Bamícori & L1 & 299 & 431 & 611 & 752 & 896 & 1248 & 1407 \\
\hline
\end{tabular}

de $\eta$. En seguida, a partir de sus soluciones inversas, expresiones 20, 28 y 34, se obtuvieron los valores de $\hat{x}$. para obtener los $E E A$, expuestos en la tabla 7 en las columnas con designación "L1, L2, L3 y L4".

\section{Análisis de los resultados}

En la tabla 7, para cada registro procesado se tienen cinco resultados del EEA, en cada FDP; es decir, 300 resultados. Se encontraron diez casos de valores iguales, por ejemplo en Choix, Huites y Chinipas en cada FDP; se adoptó el ajuste que condujo a las predicciones más elevadas. Se indicó con paréntesis circular el valor mínimo de cada FDP y con paréntesis rectangular el menor de los tres mínimos. Se observa que todos los mínimos extremos ocurren con la distribución PAG. Por lo anterior, las predicciones mostradas en la tabla 8 corresponden a tal FDP y al orden $\eta$ de momentos $L$ que condujo al EEA mínimo extremo.

El método de los momentos LH con la distribución GVE, condujo a menores $E E A$ en $7,2,1$ y 3 registros con L1, L2, L3 y L4, respectivamente (ver último renglón de la tabla 7). En cambio, con la función LOG fueron 7, 1 y
3 registros con L1, L3 y L4 y con la distribución PAG, 8, 5 y 2 registros dieron el menor $E E A$ con los momentos L1, L2 y L4, respectivamente. Es decir que con estas dos FDP el ajuste L2 y L3 no condujo a ningún valor menor del EEA.

Un contraste individual entre las predicciones mostradas en la tabla 8 y las adoptadas por Campos-Aranda (2014), procedentes de las distribuciones GVE, LOG y Log-Pearson tipo III mostró una similitud alta en todos los resultados hasta el periodo de retorno de 100 años, pero también destacó que la distribución PAG aporta, en varios registros, predicciones menores en los periodos de retorno elevados (>100 años). Por lo anterior, se estimó el llamado error relativo del $E E A$, calculado con la expresión siguiente

$E R=\frac{E E A_{M}-E E A_{m}}{E E A_{m}} 100$

en la cual, $E E A_{M}$ es el error estándar de ajuste más grande de los tres mínimos y $E E A_{m}$ es el mínimo extremo. En la última columna de la tabla 7 se observa que los $E R$ son 
menores de $10 \%$ en 11 de los 20 registros procesados y en otros siete registros no excede de $40 \%$. Entonces, los ajustes de las funciones GVE y LOG deben realizarse bajo precepto. En los dos registros en que resulta elevado el $E R$, Zopilote y Chico Ruiz, estos no presentan datos extremos elevados, sino lo contrario, pues definen parámetros de forma positivos $(k>0)$ en las tres distribuciones, entonces el ajuste con momentos LH es improcedente y ello se comprueba al encontrar EEA mínimos con los momentos L (ver columna L0 de la tabla 7).

\section{Conclusiones}

Con base en el error estándar de ajuste (EEA), los resultados de la tabla 7 indican que la distribución Pareto Generalizada condujo a las magnitudes menores de este indicador cuantitativo de la calidad estadística del ajuste, frente a las funciones GVE y LOG; debido a ello, se recomienda probarla sistemáticamente en los AFC y de otros datos hidrológicos extremos de nuestro país.

De acuerdo con los EEA mínimos que tienen errores relativos menores de $10 \%$, en la mitad de los 20 registros procesados y en otros ocho registros no exceden $40 \%$ (ver última columna de la tabla 7 ), se considera conveniente seguir aplicando las distribuciones GVE y LOG bajo precepto.

Los ajustes con momentos LH en la distribución GVE aportan menores EEA en 13 registros, con la función LOG en 11 de tales series y con el modelo PAG en 15 registros; según se observa en el último renglón de la tabla 7. Por lo anterior, el ajuste con el método de momentos L de orden mayor o momentos LH, es una opción conveniente para reducir el EEA y con ello alcanzar predicciones más confiables. Entonces, se sugiere utilizar tal técnica de manera rutinaria en los análisis de frecuencia de datos hidrológicos extremos, como una técnica avanzada del método de momentos L, mismo que ya es un procedimiento de uso generalizado.

\section{Agradecimientos}

Se agradecen las observaciones y correcciones de los dos árbitros anónimos, las cuales permitieron volver más explícito el trabajo y sus conclusiones.

\section{Referencias}

Benson M.A. Plotting positions and economics of engineering planning. Journal of Hydraulics Division, volumen 88 (número 6), 1962: 57-71.

Bhuyan A., Borah M., Kumar R. Regional flood frequency analysis of north-bank of the river brahmaputra by using LH-mo- ments. Water Resources Management, volumen 24 (número 9), 2010: 1779-1790.

Campos-Aranda D.F. Verificación de la homogeneidad regional mediante tres pruebas estadísticas. Tecnología y Ciencias del Agua, volumen 1 (número 4), 2010: 157-165.

Campos-Aranda D.F. Descripción y aplicación de la versión corregida del Test de Langbein para verificar homogeneidad regional. Ingeniería. Investigación y Tecnología, volumen XIII (número 4), 2012: 411-416.

Campos-Aranda D.F. Análisis regional de frecuencia de crecientes en la Región Hidrológica Núm. 10 (Sinaloa), México. 2: contraste de predicciones locales y regionales. Agrociencia, volumen 48 (número 3), 2014: 255-270.

Davis P.J. Gamma Function and related functions. Chapter 6, pp: 253296, en: Handbook of Mathematical Functions, Abramowitz M. \& Stegun I.A. (eds.), Dover Publications, Nueva York, 1965, 1046 p.

Fill H.D. y Stedinger J.R. Homogeneity test based upon Gumbel distribution and a critical appraisal of Dalrymple's test. Journal of Hydrology, volumen 166 (numeros 1-2), 1995: 81-105.

Gheidari M.H.N. Comparison of the L- and LH-moments in the selection of the best distribution for regional flood frequency analysis in Lake Urmia Basin. Civil Engineering and Environmental Systems, volumen 30 (número 1), 2013: 72-84.

Greenwood J.A., Landwehr J.M., Matalas N.C., Wallis J.R. Probability weighted moments: definition and relation to parameters of several distributions expressible in inverse form. Water Resources Research, volumen 15 (número 5), 1979: 1049-1054.

Hosking J.R.M. L-moments: analysis and estimation of distributions using linear combinations of order statistics. Journal of the Royal Statistical Society. B, volumen 52 (número 1), 1990: 105-124.

Hosking J.R.M. y Wallis J.R. Regional Frequency Analysis. An approach based on L-moments. Chapter 2: L-moments, pp. 14-43, chapter 5: Choice of a frequency distribution, pp. 73-86 and Appendix: L-moments for some specific distributions, pp. 191-209, Cambridge University Press, Cambridge, Inglaterra, 1997, 224 p.

IMTA (Instituto Mexicano de Tecnología del Agua). Banco Nacional de Datos de Aguas Superficiales (BANDAS), 8 CD's, CONAGUA-SEMARNAT-IMTA, Jiutepec, Morelos, 2002.

Kite G.W. Frequency and risk analyses in hydrology. Chapter 12: Comparison of frequency distributions, pp. 156-168, Water Resources Publications, Colorado, 1977, 224 p.

Lee S.H. y Maeng S.J. Comparison and analysis of design floods by the change in the order of LH-moment method. Irrigation and Drainage, volumen 52 (número 3), 2003: 231-245.

Meshgi A. y Kalili D. Comprehensive evaluation of regional flood frequency analysis by L- and LH-moments. I. A re-visit to regional homogeneity. Stochastic Environmental Research and Risk Assessment, volumen 23 (número 1), 2009a: 119-135.

Meshgi A. y Kalili D. Comprehensive evaluation of regional flood frequency analysis by L- and LH-moments. II. Development of LH-moments parameters for the generalized Pareto and 
generalized Logistic distributions. Stochastic Environmental Research and Risk Assessment, volumen 23 (número 1), 2009b: 137-152.

Moisello U. On the use of partial probability weighted moments in the analysis of hydrological extremes. Hydrological Processes, volumen 21 (número 10), 2007: 1265-1279.

Rao A.R. y Hamed K.H. Flood frequency analysis. Chapter 9: The Logistic Distribution, pp. 291-321. CRC Press, Boca Raton, USA, 2000, 350 p.

SRH (Secretaría de Recursos Hidráulicos). Boletín Hidrológico, Núm. 36. Tomos I y VI, Región Hidrológica Núm. 10 (Sinaloa), Dirección de Hidrología, México, DF, 1975.

Stedinger J.R., Vogel R.M., Foufoula-Georgiou E. Frequency analysis of extreme events. Chapter 18, pp. 18.1-18.66, en: Handbook of Hydrology, editor David R, Maidment, McGrawHill, Inc., Nueva York, 1993.

Wang Q.J. Using higher probability weighted moments for flood frequency analysis. Journal of Hydrology, volumen 194 (números 1-4), 1997a: 95-106.

Wang Q.J. LH moments for statistical analysis of extreme events. Water Resources Research, volumen 33 (número 12), 1997b: 2841-2848.

\section{Este artículo se cita: \\ Citación estilo Chicago}

Campos-Aranda, Daniel Francisco. Ajuste de las distribuciones GVE, LOG y PAG con momentos L de orden mayor. Ingeniería Investigación y Tecnología, XVII, 01 (2016): 131-142.

\section{Citación estilo ISO 690}

Campos-Aranda D.F. Ajuste de las distribuciones GVE, LOG y PAG con momentos $\mathrm{L}$ de orden mayor. Ingeniería Investigación y Tecnología, volumen XVII (número 1), enero-marzo 2016: 131-142.

\section{Semblanza del autor}

Daniel Francisco Campos-Aranda. Obtuvo el título de ingeniero Civil en diciembre de 1972, en la entonces Escuela de Ingeniería de la UASLP. Durante el primer semestre de 1977, realizó en Madrid, España un diplomado en hidrología general y aplicada. Posteriormente, durante 1980-1981 llevó a cabo estudios de maestría en ingeniería en la especialidad de Hidráulica, en la División de Estudios de Posgrado de la Facultad de Ingeniería de la UNAM. En esta misma institución, inició (1984) y concluyó (1987) el doctorado en ingeniería con especialidad en aprovechamientos hidráulicos. Ha publicado trabajos principalmente en revistas mexicanas de excelencia: 50 en Tecnología y Ciencias del Agua (antes Ingeniería Hidráulica en México), 18 en Agrociencia y 18 en Ingeniería. Investigación y Tecnología. Es profesor jubilado de la UASLP, desde el $1^{\circ}$ de febrero de 2003. En noviembre de 1989 obtuvo la medalla Gabino Barreda de la UNAM y en 2008 le fue otorgado el Premio Nacional "Francisco Torres H." de la AMH. A partir de septiembre de 2013 vuelve a ser investigador nacional nivel I. 